\title{
Mobilidata: Um sistema para análise do transporte coletivo municipal
}

\author{
Tatiele M. Razera, Ademir Camillo Junior, Sandro A. Janotte, Luís P. da Silva, \\ Roberto A. Faustinelli
}

Faculdade Senai - Serviço Nacional de Aprendizagem Industrial de Santa Catarina R. Arno Waldemar Dohler, 957 - Zona Industrial Norte - 89219-510 - Joinville - SC

$\{$ tatiele.razera, ademir.camillo\} @edu.sc.senai.br, \{sandro_janotte,
luis_paulo, roberto_faustinelli\} destudante.sc.senai.br

\begin{abstract}
Municipal public management is a complex task, which requires specialists and technologies to assist in municipal management. In the area of mobility, the difficulties are more and more visible every day, due to the increase of the population and consequently vehicles in circulation. In order to contribute with traffic specialists, Mobilidata was created, a system that allows the analysis of the municipal (public) public transport, from the tool it is possible to verify localities with lack of transport, as well as times and places with greater circulation of vehicles for public transport, among other functionalities to help managers in the verification of demands and problems of urban transport in the municipality.
\end{abstract}

Resumo. A gestão pública municipal é uma tarefa complexa, a qual demanda especialistas e tecnologias de auxílio na gestão municipal. Na área de mobilidade, as dificuldades estão cada dia mais visiveis, devido ao aumento da população e consequentemente veículos em circulação. De forma a contribuir com especialistas de trânsito, foi criado o Mobilidata, um sistema que permite a análise do transporte coletivo (público) municipal, a partir da ferramenta é possivel verificar localidades com carência de transporte, além dos horários e locais com maior circulação de veículos destinados ao transporte coletivo, entre outras funcionalidades, para auxiliar os gestores na verificação de demandas e problemas do transporte urbano no município.

\section{Introdução}

A mobilidade vêm sendo um dos grandes desafios para gestores públicos, principalmente em áreas urbanas, onde a população está crescendo, sendo que no Brasil a população das áreas urbanas no ano de 2015 chegava em $84 \%$, sendo a região sul com um total de $86 \%$, de acordo com o [Instituto Brasileiro de Geografia, 2019].

Esse aumento populacional ocorreu por diferentes fatores, como, o êxodo rural, ocasionado devido a mecanização da produção agrícola, nas décadas de 1970 e 1980, gerando um processo chamado de metropolização (ocupação urbana que ultrapassa os limites das cidades), [Instituto Brasileiro de Geografia, 2019].

Devido ao crescimento habitacional em áreas urbanizadas a população acabou sendo distribuída nas diferentes regiões das cidades, o que gerou um aumento considerável na quantidade de moradores em regiões periféricas, sendo estas regiões atrativas por classes sociais mais baixas e que dependem do transporte público, fazendo com que as soluções para esse tipo de locomoção não conseguisse acompanhar o 
crescimento populacional, gerando impactos sociais e ambientais, [de Carvalho e Pereira, 2011].

A pesquisa sobre a Mobilidade Urbana da Região Metropolitana de São Paulo, ainda mostra que na década entre 2007 e 2017, houve crescimento da mobilidade por meio de transporte coletivo entre a população de menor renda, [Secretaria dos Transportes Metropolitanos, 2017].

Além do transporte público (coletivo), a população com renda mais baixa também utiliza meios de locomoção não motorizados, como viagens a pé e bicicleta, por exemplo, chegando a existir uma diferença de $144,11 \%$ no uso de transporte não motorizado pela faixa de renda mais baixa em relação a faixa de renda mais alta, indicando uma clara tendência no uso de transporte não motorizado pela população de baixa renda, [Secretaria dos Transportes Metropolitanos, 2017].

Além da renda, fatores como, preço da condução, pontos/estações distantes e a baixa frequência da condução na sua região, tornam o transporte não motorizado mais atrativo, [Gomide, 2003].

[De Carvalho et al., 2013] afirmam que um dos motivos para o aumento das tarifas e ônibus nos últimos anos está relacionado aos congestionamentos, reflexo da grande preferência que existe hoje pelo transporte individual, o qual é agravado por conta da insatisfação com o transporte público.

Segundo o [Ministério dos Transportes, Portos e Aviação Civil, 201?], ocorreu um aumento de 42,94\% na frota de automóveis do sul do Brasil entre 2010 a 2017.

Analisando os dados levantados pelo índice de tráfego da empresa [Tomtom, 2019], é possível ver um aumento nos níveis de congestionamento em Porto Alegre e Curitiba de 2017 para 2018. Devido a esse aumento nos congestionamentos, a qualidade do transporte público acaba sendo afetada, diminuindo assim a sua demanda, que por sua vez gera aumento das tarifas em uma tentativa de reequilibrar as receitas e custos, [de Carvalho e Pereira, 2011].

Seguindo as ocorrências das grandes cidades, a população de Joinville (SC) também demonstra sua insatisfação com o transporte público, optando cada vez mais por meios de locomoção particulares. Como pode ser visto na pesquisa Cidade em Dados, do ano de 2019 realizada pela Prefeitura Municipal da cidade, a qual analisou os dados entre os anos de 2007 e 2017 e observou que houve uma queda de 43,66\% no uso de transporte público e um aumento de $70.68 \%$ no uso de transporte particular, [Secretaria de Planejamento Urbano e Desenvolvimento Sustentável, 2019].

Além do crescimento do uso de transporte privado, Joinville também segue a tendência das demais cidades quando o assunto é distribuição da população em relação a renda.

Com base nos dados disponibilizados na revista Joinville Bairro a Bairro 2017, [Secretaria de Planejamento Urbano e Desenvolvimento Sustentável, 2017], ao ser realizada uma comparação entre a renda média e a distância do centro ao bairro, dividido em 5 faixas de distância ao centro e agrupando os resultados dentro de cada faixa, percebe-se que os bairros que estão na primeira faixa $(0 \mathrm{~km}$ a $3,298 \mathrm{~km})$, possuem uma renda média $247,98 \%$ maior do que a última faixa $(13,192 \mathrm{~km}$ a $16,490 \mathrm{~km})$. Estes dados corroboram com as informações de, [de Carvalho e Pereira, 2011]. 
[D’Agosto, 1999] aponta a priorização do transporte público como uma providência necessária para a melhoria no desempenho dos sistemas de transportes, porém, também avalia que para isso é necessária uma evolução na forma que os transportes públicos atuam.

Segundo [Gomide, 2003] a baixa frequência de condução e distância entre paradas, são dois dos três maiores problemas do transporte público.

Visando a melhoria do transporte coletivo municipal o objetivo deste projeto é desenvolver uma aplicação web que possibilite a visualização gráfica de dados relacionados aos pontos de parada, itinerários e cobertura do transporte público, para tal foram utilizados os dados do transporte coletivo da cidade de Joinville, Santa Catarina, no ano de 2018.

Essa aplicação deverá ser acessada por meio de softwares navegadores e será destinada para especialistas de trânsito e gestores públicos de forma a colaborar em análises e necessidades da cidade, relacionadas ao transporte público, buscando assim melhorias no sistema de transporte público coletivo.

A partir dos dados fornecidos pelo MobiliData espera-se ser possível oferecer soluções para a diminuição do tempo de espera nas paradas e de viagem nos percursos, bem como o melhor posicionamento de paradas de onibus, reduzindo o tempo de caminhada até a mesma, o que vem a contribuir com a melhoria da qualidade de vida dos usuários de transporte coletivo.

Com o aplicativo proposto será possível visualizar todas as linhas de ônibus que passam em determinadas paradas, permitindo a filtragem por horário e visualização das distâncias entre os pontos de paradas de ônibus. Também será possível a filtragem por linha de ônibus, possibilitando a visualização do percurso.

\section{Trabalhos Relacionados}

O artigo Busão: um Sistema de Informações Móvel para Auxílio à Mobilidade Urbana Através do Uso de Transporte Coletivo apresenta um sistema de informações móvel, que visa apoiar os usuários de ônibus, fornecendo informações em tempo real sobre os ônibus disponíveis na cidade, através de técnicas de geoprocessamento baseadas na localização dos usuários, pontos de interesse e rotas dos ônibus [Leite et al., 2018].

$\mathrm{Na}$ dissertação de [D’Agosto, 1999] apresentou diversas variáveis a serem analisadas ao avaliar o desempenho de um sistema de transporte urbano.

[Gomide, 2006] faz uma análise voltada para a desigualdade social em transportes públicos e os impactos negativos dessa falta de acessibilidade das pessoas com faixas de rendas mais baixas ao transporte público.

[de Carvalho e Pereira, 2011] analisam o aumento contínuo das tarifas de transportes públicos e a queda constante da renda da população brasileira, destacando a relação de ambos e demonstrando um ciclo vicioso.

[Françoso, de Mello, 2016] pesquisam os impactos positivos e negativos no constante desenvolvimento e avanço de aplicativos para smartphones capazes de monitorar e criar rotas para o transporte público e privado, os aplicativos focados na pesquisa utilizam o sistema de georreferenciamento. 
No artigo [de Coelho et al, 2016] é realizada uma pesquisa para descobrir o perfil dos usuários da Uber (empresa tecnológica de serviço individual de transporte) e as principais motivações dos mesmos para utilizar o aplicativo.

\section{Métodos}

O projeto foi iniciado com um estudo sobre o funcionamento do transporte público, quais seriam as informações necessárias e se havia alguma padronização para essas informações, com isso descobrimos que na grande maioria as aplicações, utilizam modelos similares aos criados pela Google Maps, que é bem completo e organizado.

Em seguida foram realizadas as coletas dos dados dos itinerários, paradas e horários do transporte coletivo no site www.onibus.info, para isso foi desenvolvida uma ferramenta automatizada.

Com os dados prontos, foram realizados testes de desempenho para ferramentas de exibição das paradas sobre os mapas, nesta etapa foram testados o Google Maps, Here Maps e Open Street Maps, sendo que o Here Maps apresentou o melhor desempenho, exibindo o mapa com mais agilidade independente da quantidade de requisições enviadas, assim como a possibilidade de uso gratuíto da ferramenta.

O projeto MobiliData foi dividido em dois subsistemas, o primeiro chamado de Back-end, que é a aplicação a qual fica no servidor, sem a interação direta do usuário, porém o responsável pela execução das regras de negócio. O segundo é o Front-end, que é interface visual exibida em um navegador web, na qual o usuário realiza suas interações com a aplicação.

O Back-end foi desenvolvido com a linguagem de programação Java, utilizando o Eclipse que é uma IDE (Integrated Development Environment) ou Ambiente de Desenvolvimento Integrado por ser muito conhecido, de fácil utilização e possuir muitos plugins e subsistemas que podem ser adicionados para prover recursos necessários no funcionamento da aplicação, [Eclipse Foundation, 2019].

A linguagem Java foi escolhida por ser uma linguagem de programação orientada a objeto, rápida, segura e confiável, que foi projetada para permitir o desenvolvimento de aplicações portáteis de alto desempenho para a mais ampla variedade de plataformas de computação, [Oracle Corporation, 2019].

Também foi utilizado o framework Spring, o qual foi escolhido pelo fato de proporcionar muitas configurações automáticas, liberando-nos para um desenvolvimento focado nas questões de negócios ou áreas afins, [Foncesa Junior, 2007]. O Spring também provê o suporte necessário para persistência e manipulação dos registros no banco de dados, que é o local onde os dados da aplicação são armazenados, [Foncesa Junior, 2007].

Para banco de dados foram utilizados o MySQL no armazenamento dos cadastro para garantir o relacionamento das entidades e o banco de dados MongoDB por realizar as consultas mais rapidamente que o MySQL.

O Back-end foi desenvolvido como RestFull API (Application Programming Interface) ou seja, Interface de Programação de Aplicativos, que é uma técnica utilizada para proporcionar disponibilidade de acesso ao aplicativo a partir de outros sistemas independentes, sem manter sessão de usuário, através da geração de Tokens. 

Token).

Para autenticação utilizou-se o padrão OAuth2 com Token JWT (Json Web

O conceito da autenticação OAuth2 com token JWT está no usuário enviar sua solicitação de autenticação para o servidor, que valida a conta e senha enviada e identifica os papéis permitidos para este usuário, gera um Token com essas informações e permissões e encaminha ao cliente que irá armazená-lo. Ao realizar requisições o cliente reenvia o Token que armazenou para o servidor, onde são analisadas e recuperadas as informações. O Token é configurado com um tempo de vida pré-estabelecido, quando esse tempo é expirado obriga o cliente a fazer nova autenticação, caso o usuário saia do aplicativo, o Token é eliminado dos Cookies do navegador do cliente.

Na programação do Front-end, optou-se pelo Visual Studio Code, que é um editor de código-fonte leve e poderoso, disponível para Windows, macOS e Linux, [Microsoft, 2018].

O Node.js oferece suporte às linguagens de programação JavaScript, TypeScript e o ambiente de execução para aplicações Javascript fora do navegador, [OpenJS Foundation, 2018], contribuindo assim no desenvolvimento e na utilização do Angular, que é um framework e plataforma de desenvolvimento de interfaces para aplicações $\mathrm{Web}$ e Mobile, baseado em HTML, CSS e TypeScript.

O Angular segue o modelo SPA (Single Page Application) que exibe a aplicação em uma única página, onde são carregados e descarregados os componentes, conforme interações do usuário e sem a necessidade da recarga de todo conteúdo da página, que resulta em melhora significativa de desempenho e diminuição de tráfego de dados entre servidor e cliente, [Google, 2018b].

Para a estilização da aplicação, o Angular Material fornece um conjunto de componentes de interface do usuário reutilizáveis, bem testados e acessíveis, baseado no Material Design da Google. Componentes padronizados proporcionam a criação de um estilo bem definido e harmônico, excelente apresentação e ótima usabilidade, [Google, 2018a].

O Here Maps é um serviço de posicionamento guiado por satélite semelhante ao Google Maps, sua principal finalidade é facilitar a locomoção das pessoas para chegar a um destino desejado, seja caminhando, deslocando-se de bicicleta, automóvel, transporte público ou outro meio, [Here Technologies, 2018].

Segundo [Here Technologies, 2018] sua visão é: “A era do mundo autônomo”. A localização é tudo que diz respeito à forma como olhamos aqui, para realizar nossa missão de possibilitar um mundo autônomo para todos. Ao criar uma representação digital da realidade inteiramente construída sobre dados de localização, estamos transformando radicalmente a maneira como o mundo vive, se move e interage, [Here Technologies, 2018].

A aplicação de Front-end foi projetada para exigir autenticação de cliente com dois níveis de acesso: Administrador e Usuário, e dois módulos: Mapas e Usuários.

Ao autenticar-se no MobiliData com nível de administrador, o utilizador terá acesso completo aos módulos. Quando autenticado com nível de usuário, terá acesso somente ao módulo Mapas. O módulo Usuários é o local onde são realizados os cadastros e alterações de usuários para acesso ao aplicativo. 
O módulo Mapas possui seis tipos de exibições distintas, onde é possível aplicar filtros para dias úteis, sábados e domingos, intervalo de horários, endereço e bairros em todos os mapas, com exceção do mapa de rotas das linhas. São elas:

- Paradas, que exibe todas as paradas de ônibus armazenadas, ao clicar em um marcador de parada serão exibidos os dados de endereço, latitude e longitude;

- Agrupadas, destinado a exibir um contador de paradas agrupadas em uma determinada região, é incrementado ou decrementado ao realizar zoom sobre o mapa;

-Viagens, apresenta as paradas no mapa, que ao serem selecionadas exibem linhas que fazem parte da parada e total de viagens que são feitas por período na mesma;

- Linhas, que exibe as paradas no mapa e ao selecionar uma, serão exibidas as linhas que fazem parte da parada;

- Cobertura, imprime as paradas em duas camadas de cores que permitem uma análise visual de distância entre paradas, a cor amarela tem finalidade de mostrar se a distância entre paradas está dentro de critérios considerados aceitáveis e a cor verde dentro do ideal;

- Rotas, ao selecionar uma linha de ônibus são traçadas as rotas de ida e volta sobre o mapa;

Após seguir essa sequência de tarefas, conseguimos desenvolver uma aplicação que atende as necessidades apresentadas até então. A seguir na seção de resultados, apresentamos o aplicativo desenvolvido, com suas telas e filtros.

\section{Resultados}

Em todas as telas desenvolvidas é exibido o menu superior, em cor cinza escuro, o qual apresenta os itens da esquerda para a direita, MobiliData (página inicial), Mapas e Usuários e no lado direito do тепи o botão Sair, como pode ser observado na Figura 1.

Na Figura 1 está sendo apresentada a tela de listagem de usuários, onde é possível fazer consultas, inclusões, alterações e exclusões de usuários. Ao realizar uma consulta é possível filtrar os resultados por Nome, Email ou Status utilizando os filtros localizados na parte superior da tela, logo após o título Usuários.

Para inserir novos usuários, deve-se clicar no botão Novo Usuário, em seguida será apresentada uma nova tela solicitando os dados para inserção. Para editar ou excluir, deve-se clicar no botão desejado da coluna Ações, localizado ao fim da linha na tabela de exibição dos usuários. Estas ações podem ser observadas ainda na Figura 1.

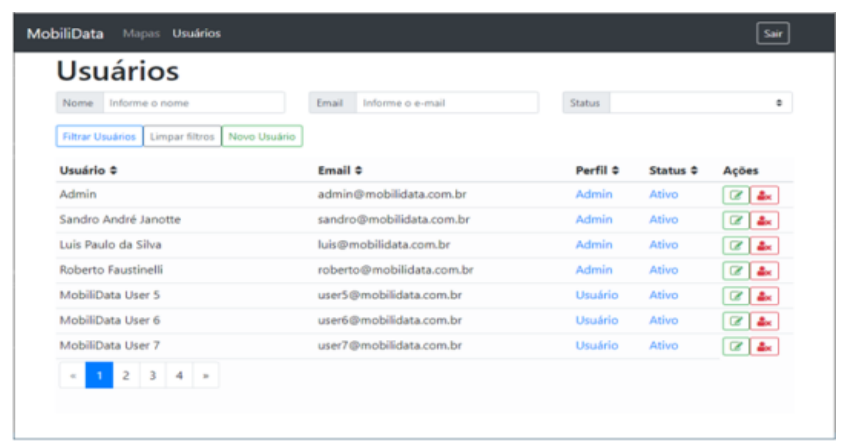

Figura 1: Lista de usuários do aplicativo 
Ao selecionar Mapas no menu principal é exibido o submenu com as visualizações disponíveis, são elas: Paradas, Agrupadas, Viagens, Linhas, Cobertura e Rotas.

$\mathrm{Na}$ Figura 2 são apresentadas as paradas de ônibus da cidade de Joinville, sendo possível ampliar o mapa para melhor visualização. Ao clicar em uma parada são exibidas as informações referentes a mesma. Também é possível filtrar os resultados por dia (útil, sábado e domingo), endereço, bairro e por faixa de horários, como na Figura 2, que está filtrada por dias úteis, das 07:00 às 09:00.

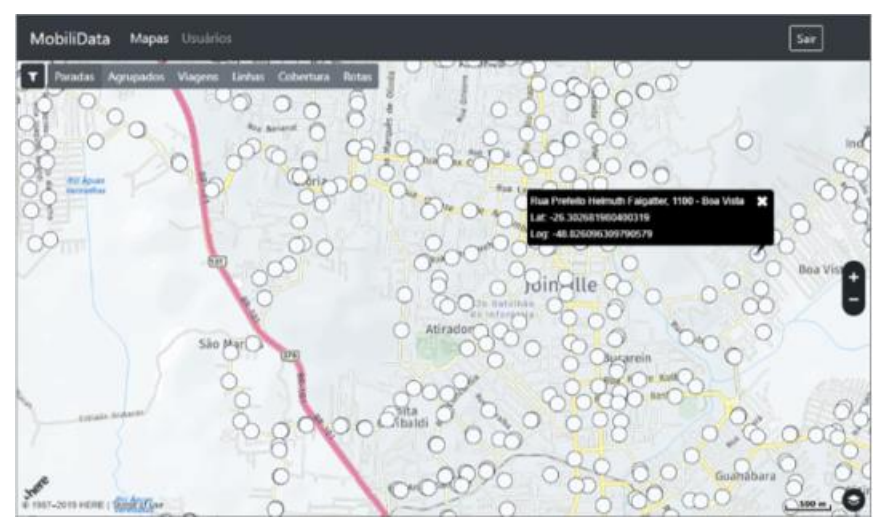

Figura 2: Paradas do transporte público de Joinville

Na Figura 3 são apresentadas as mesmas paradas, porém com a exibição de mapa de paradas Agrupadas. $\mathrm{O}$ agrupamento mostrado na Figura 3 faz a união das paradas por região, exibindo um contador de pontos de parada, este contador é incrementado ou decrementado de acordo com o zoom de visualização do mapa. Ao aumentar o zoom no mapa são exibidos mais detalhes do mapa e o contador é diminuído, pois o agrupamento diminui detalhes da região, mostrando assim menos pontos separados.

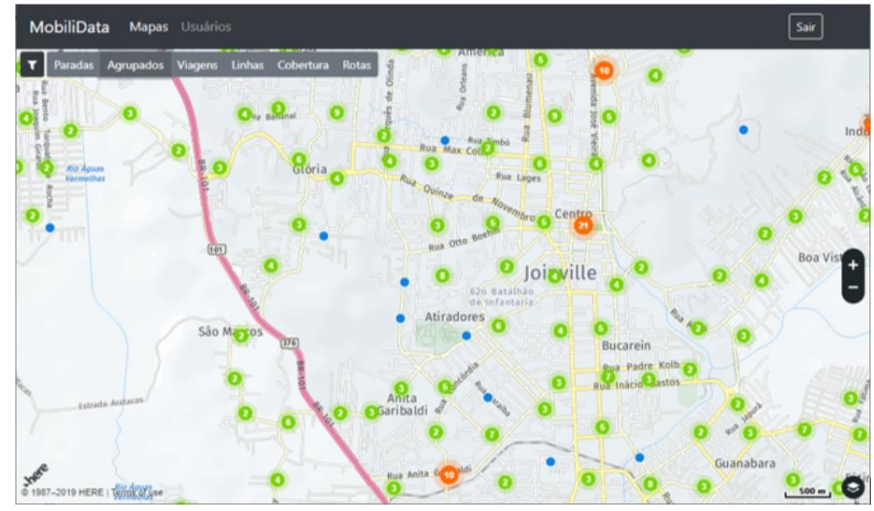

Figura 3: Mapa de paradas Agrupadas

Na Figura 4 é possível observar o Mapa de Viagens, onde cada ponto apresenta uma coloração em tons de azul, que representa a quantidade média de viagens que passam pelo ponto em questão, sendo possível verificar a quantidade relacionada com a coloração da legenda abaixo dos filtros. As cores mais escuras representam um número maior de viagens.

Ainda na Figura 4 identifica-se as informações de uma parada específica, conforme exibido na área central da imagem. Ao clicar em uma parada são exibidas as viagens e a quantidade de viagens que passam por aquele ponto. No exemplo, da Figura 
4, está sendo apresentada a Linha 0140 - Tupy/Guanabara com 9 viagens, sendo que essas viagens passam no ponto selecionado, no período e dia filtrado.

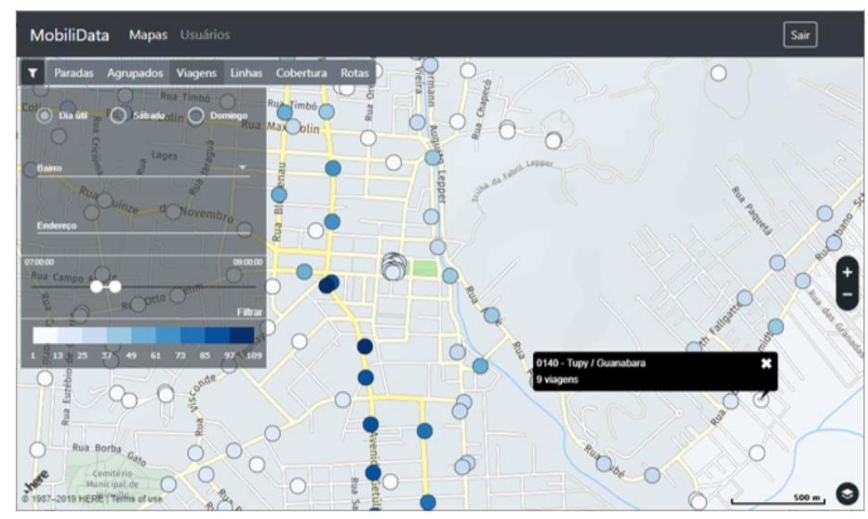

Figura 4: Mapa de Viagens filtrado por dia e horário

No Mapa de Linhas apresentado na Figura 5, assim como no Mapa de Viagens, as faixas têm suas legendas e cores alteradas de acordo com as informações que estão sendo apresentadas, para as linhas as cores são alteradas seguindo tons de vermelho que seguem o mesmo padrão, as cores mais escuras representam maior número de linhas passando pelo ponto. As informações das linhas, também podem ser visualizadas clicando sobre um ponto de parada.

Como pode ser visto na Figura 5, na parada selecionada passam 3 linhas, analisando-se a cor do ponto selecionado em comparação com a legenda, assim como pode-se visualizar na janela aberta as linhas que passam na parada ao clicar sobre a mesma, neste caso, 0040 - Tupy/Norte via Centro, 0043 - Tupy/Centro e 0140 Tupy/Guanabara.

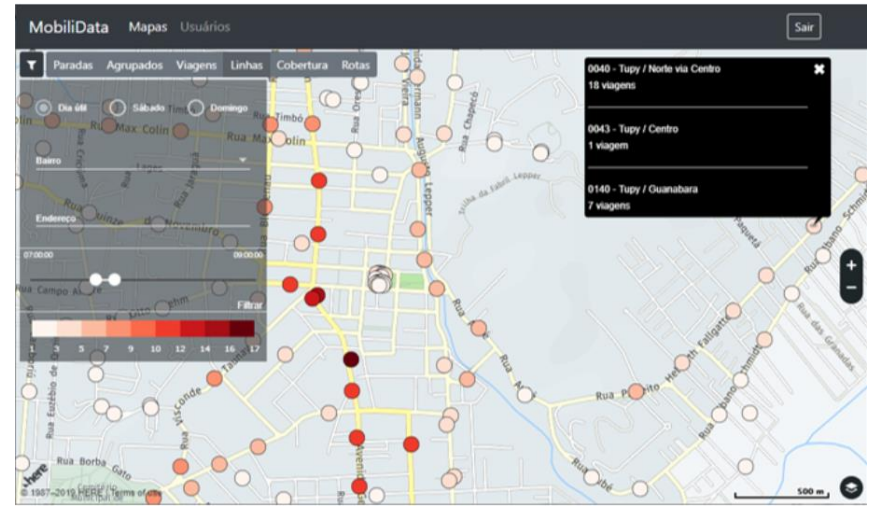

Figura 5: Mapa de Linhas filtrado por dia e horário

A cobertura das paradas de ônibus pode ser vista na Figura 6, as paradas são exibidas com dois marcadores circulares de perímetro distintos, o marcador amarelo indica distâncias aceitáveis, enquanto o verde indica distâncias ideais entre pontos.

A distância aceitável, amarela é de 300 e 500 m e a distância ideal, verde igual ou inferior $300 \mathrm{~m}$. Áreas sem marcações apresentam regiões com distâncias superiores a 500 $\mathrm{m}$, que são consideradas ruins para a acessibilidade e qualidade do transporte público, [Foncesa Junior, 2011]. 


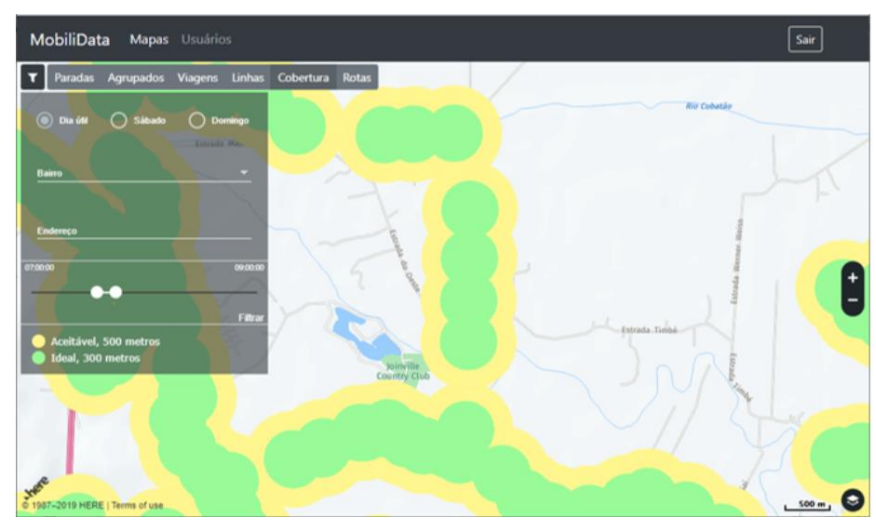

Figura 6: Cobertura de área de paradas

Como pode ser visto na Figura 6, a cidade de Joinville ainda apresenta muitas regiões com paradas distantes, situação essa que pode ser melhorada ao analisar a distribuição de pontos no mapa, como proposto neste trabalho.

Ao acessar o Mapa de Rotas e selecionar uma das linhas, como pode-se ver na Figura 7. as rotas de ida e de volta são traçadas no mapa, bem como as respectivas paradas de ônibus. Também é possível verificar informações como endereço, latitude e longitude de cada parada clicando sobre ela.

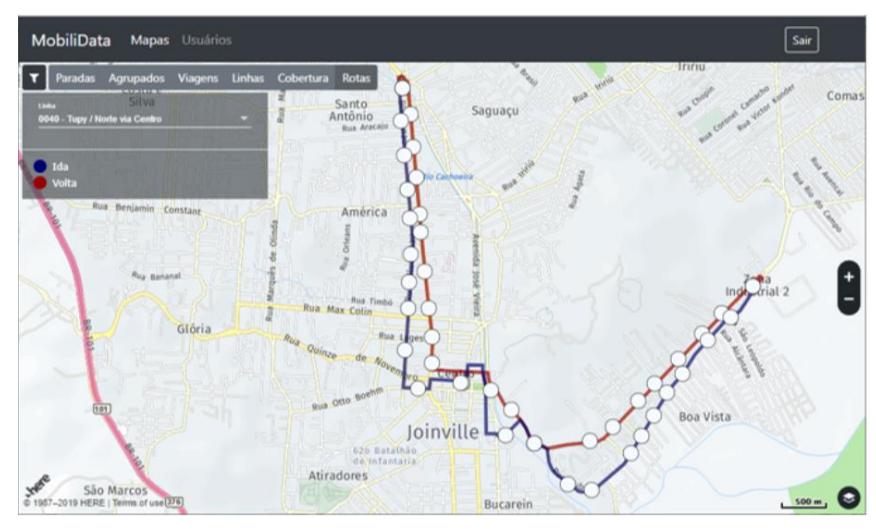

Figura 7: Mapa de rotas das linhas

Como pode ser observado nas Figuras de 2 a 7 , o sistema apresenta diferentes informações para análise do transporte coletivo, possibilitando assim que gestores e especialistas de trânsito tenham a possibilidade de verificar melhorias na qualidade do transporte e assim contribuir com melhorias na qualidade da mobilidade das cidades.

\section{Considerações Finais}

O objetivo deste projeto consiste em apresentar os dados de transporte coletivo, utilizando como base os dados da cidade de Joinville/SC, de forma que os gestores, especialistas e empresários responsáveis por esse meio de locomoção tenham a oportunidade de oferecer melhorias à população.

Com o uso da ferramenta, as melhorias podem ocorrer através da manutenção de distribuição dos horários, pontos de parada e remanejamento de trajetos de linhas, fatores estes que quando mal gerenciados acarretam em problemas para o sistema de transporte público coletivo, gerando superlotação, aumento do tempo das viagens e grandes distâncias percorridas pelos usuários até o ponto. 
Ao visualizar os dados referentes ao transporte público, de forma gráfica e ainda com as opções de filtros e exibição em mapas, os gestores têm a oportunidade de oferecer soluções mais precisas para o transporte coletivo da cidade, o que pode gerar maior interesse dos usuários por esse tipo de locomoção, diminuindo outros problemas para a cidade, como os congestionamentos, que vem aumentando ano a ano, devido ao maior interesse da população por meios de transportes individuais.

Também é possível verificar a distribuição de linhas e horários em toda a cidade, podendo assim oferecer soluções adequadas para a população das áreas mais distantes da região central, que como demonstrado, apresentam maior necessidade do transporte coletivo.

Como trabalhos futuros sugere-se testar a aplicação com dados do transporte coletivo de outras cidades, assim como disponibilizar opções para inserir, editar ou excluir linhas, paradas, rotas e horários de forma a possibilitar simulações de melhorias do transporte coletivo.

\section{Referências}

Coelho, L. A. d. A., Silva, L. A. d. S., de Andrade, M. O.and Maria, M. L. A. (2016). Perfil socioeconômico dos usuários da uber e fatores relevantes que influenciam a avaliação desse serviço no brasil, Congresso Nacional de Pesquisa em Transporte da Associação Nacional de Pesquisa e Ensino em Transportes XXXI: 1-9. Disponível em: http://146.164.5.73:30080/tempsite/anais/documentos/2017/Aspectos\%20Econo micos\%20Sociais\%20Politicos\%20e\%20Ambientais\%20do\%20Transporte/Regulaca o\%20em\%20Transportes\%20II/5_498_AC.pdf

D’Agosto, M. d. A. (1999). Avaliação do desempenho operacional de sistemas de transportes urbanos em vias segregadas, Mestrado em ciências em engenharia de transportes, Mestrado em Ciências em Engenharia de Transportes.

de Carvalho, C. H. R., Gomide, A., Pereira, R. H. M., Mation, L. F., Balbim, R., Neto, V. C. L., Pereira, G. E., Krause, C. and Guedes, E. P. (2013). Nota Técnica:Tarifação e financiamento do transporte público urbano, Instituto de Pesquisa Econômica Aplicada. Disponível em: http://repositorio.ipea.gov.br/bitstream/11058/1365/1/Nota Tecnica_Tarifa $\% \mathrm{C} 3 \% \mathrm{~A} 7 \% \mathrm{C} 3 \% \mathrm{~A} 30 \_$e financiamento_do_transporte_p $\% \mathrm{C} 3 \% \mathrm{BAb}$ lico_urbano.pdf.

de Carvalho, C. H. R. and Pereira, R. H. M. (2011).Efeitos da variação da tarifa e da renda da população sobre a demanda de transporte público coletivo urbano no brasil, Texto para Discussão 1595: 7-8. Disponível em: http://repositorio.ipea.gov.br/bitstre am/11058/1282/1/TD_1595.pdf.

Eclipse Foundation (2019). Eclipse IDE, The Leading Open Platform for Professional Developers. Disponível em: https://www.eclipse.org/eclipseide.

Foncesa Junior, J. R. N. (2007). Estudo de caso usando o spring framework para criação de aplicações web com j2ee, Especialização em sistemas de computação para web, Mestrado em Ciências em Engenharia de Transportes.

Foncesa Junior, J. R. N. (2011). Análise da acessibilidade do sistema de transporte público urbano. estudo de caso na cidade de são carlos - sp, Mestrado em ciências, 
programa de pós graduação em engenharia de transportes, Escola de Engenharia de São Carlos da Universidade de São Paulo.

Françoso, M. T. and de Mello, N. C. (2016). Influência dos aplicativos de smartphones para transporte urbano no trânsito, Congresso Luso Brasileiro para o Planejamento Urbano, Regional, Integrado e Sustentável 7. Disponível em: http://www.fau.ufal.br/evento/pluris2016/files/Tema\%202\%20-

\%20Cidades\%20Inovadoras\%20e\%20\%20Inteligentes/Paper800.pdf.

Gomide, A. d. A. (2003). Transporte urbano e inclusão social: elementos para políticas públicas, Texto para Discussão 960: 7-16. Disponível em: http://repositorio.ipea.gov. br/bitstream/11058/1282/1/TD_1595.pdf.

Gomide, A. d. A. (2006). Mobilidade urbana, iniquidade e políticas sociais, Instituto de Pesquisa Econômica Aplicada. Disponível em: http://repositorio.ipea.gov.br/bitstrea m/11058/4511/1/bps_n.12_ensaio5_alexandre12.pdf.

Google (2018a). Angular Material: Material Design Components for Angular. Disponível em: https://v6.material.angular.io.

Google (2018b). Introduction to the Angular Docs. https://angular.io/docs/.Instituto Brasileiro de Geografia (2019). Efeitos da variação da tarifa e da renda da população a demanda de transporte público coletivo urbano no Brasil, Instituto Brasileiro de Geografia. Disponível em: https://educa.ibge.gov.br/jovens/conheca-o-brasil/populac ao/18313-populacao-rural-e-urbana.html.

Instituto Brasileiro de Geografia (2019). Conheça o Brasil - População. População Rural e Urbana. Disponível em: https://educa.ibge.gov.br/jovens/conheca-obrasil/populacao/18313-populacao-rural-e-urbana.html.

Leite, D. F. B., Rocha, J. H. and Baptista, C. d. S. (2018). Busão: um sistema de informações móvel para auxílio à mobilidade urbana através do uso de transporte coletivo, Simpósio Brasileiro de Sistemas de Informação XIV: 170-181. Disponível em: https://sol.sbc.org.br/index.php/sbsi/article/view/5685/5582.

Microsoft (2018). Getting Started. Disponível em: https://code.visualstudio.com/docs.

Ministério dos Transportes, Portos e Aviação Civil (201?). Anuário Estatístico de Segurança Rodoviária 2010-2017. Disponível em: https://www.infraestrutura.gov.br/ images/BIT_TESTE/Publica\%C3\%A7oes/Anuari_Estatistico_de_Seguranca_Rodovi aria.pdf.

OpenJS Foundation (2018). Node JS. Disponível em: https://nodejs.org/en/docs/.

Oracle Corporation (2019). Obtenha Informações sobre a Tecnologia Java. Disponível em: https://www.java.com/pt_BR/about.

Secretaria de Planejamento Urbano e Desenvolvimento Sustentável (2017). Joinville Bairro a Bairro 2017, Prefeitura de Joinville -SC. Disponível em: https://www.joinville.sc.gov.br/wp-content/uploads/2017/01/Joinville-Bairro-aBairro-2017.pdf.

Secretaria de Planejamento Urbano e Desenvolvimento Sustentável (2019). Joinville Cidade em Dados 2019: Ambiente Construído, Prefeitura de Joinville -SC. Disponível em: $\quad$ https://www.joinville.sc.gov.br/wp-content/uploads/2019/08/Joinville -Cidade-em-Dados-2019-Ambiente-Constru\%C3\%ADdo.pdf. 
Secretaria dos Transportes Metropolitanos (2017). A mobilidade urbana da região metropolitana de são paulo em detalhes, Pesquisa Origem Destino. Disponível em: https://transparencia.metrosp.com.br/node/1516/.

Here Technologies. (2018). Welcome to Here. Disponível em: https://www.here.com.

Tomtom (2019). Traffic Index: 2018. Disponível em: https://www.tomtom.com/en_gb/ traffic-index/ranking/? country $=\mathrm{BR}$. 